\title{
What Factors Hindered the Access to Essential Anti- Cancer Medicine in Public Hospitals amongst the Local Population in Hubei Province, China?
}

\section{Chaoyi Chen}

Huazhong University of Science and Technology

\section{Zhanchun Feng}

Huazhong University of Science and Technology

\section{Ziqi Yan}

Huazhong University of Science and Technology

\section{Yufeng Ding}

Huazhong University of Science and Technology

Jia Wang

Huazhong University of Science and Technology

\section{Ruoxi Wang}

Huazhong University of Science and Technology

\section{Qian Fu}

Huazhong University of Science and Technology

Da Feng ( $\sim$ qq328867128@126.com)

Huazhong University of Science and Technology

\section{Research Article}

Keywords: Essential Anti-Cancer Medicine, Availability, Affordability, Price, Cancer

Posted Date: March 4th, 2021

DOI: https://doi.org/10.21203/rs.3.rs-262997/v1

License: (1) (1) This work is licensed under a Creative Commons Attribution 4.0 International License. Read Full License 


\section{Abstract}

\section{Background}

Cancer poses a serious threat to one's health, which caused significant economic burden on the family and society. Poor availability and affordability resulted in some essential medicines failing to meet the basic health needs of this group of patients. The objective of this study was to evaluate the availability, prices and affordability of 32 anticancer essential medicines in Hubei Province, China.

\section{Methods}

Data on the availability and price related information of 32 essential anticancer medicines in the capital and five other cities of Hubei Province were collected. A total of 28 hospitals were sampled, which included 13 tertiary hospitals and 15 secondary hospitals. We used the standard methods developed by the World Health Organization (WHO) and Health Action International (HAl) to compare the differences in drug price, availability and affordability between secondary hospitals and tertiary hospitals.

\section{Results}

Overall, the availability of medicine was higher in tertiary hospitals. The average availability of originator brand (OBs) was $13.70 \%$ (tertiary hospitals) VS 6.67\% (secondary hospitals), and lowest-priced generic (LPGs) was $62.83 \%$ (tertiary hospitals) VS $42.92 \%$ (secondary hospitals). The MPR value of most sampled medicines in secondary hospitals were less than 1. In contrast, the MPR of Cytarabine (17.15), Oxaliplatin (12.73) were significantly higher than the international reference price. The top three OBs' total expenses for 30-day treatment were Irinotecan, Oxaliplatin, Bicalutamide. Further, their affordability was relative low, as the costs for one course using these medicines were much higher than $20 \%$ of the minimum family monthly income.

\section{Conclusion}

Though the "Zero Mark-Up" and "Two Vote" Policies have been implemented in China, the availability issue yet to be addressed. High price and low affordability were the major barriers to the access of essential anticancer medicines. Measures should be taken to provide sufficient, available and affordable medicines to patients in need.

\section{Introduction}

Cancer has become a serious health problem, which contributed to severe burden of disease and even death ${ }^{[1]}$. A total of 9.6 million deaths due to cancer diseases were reported worldwide in $2018^{[2]}$. China accounts for $21.0 \%$ of the worldwide new cancer cases, and total of 22.96 million Chinese residents died of cancer ${ }^{[3]}$. At present, anticancer medicine is a core component of treatment, but barriers to receiving cancer therapy such as high price of anticancer medicines and poor availability were yet to be solved ${ }^{[4]}$. These problems may be related to medical information asymmetry and the distribution of responsibilities 
between the drug purchasing sector and the patients who bear the cost restrict competition in the market [5].

Essential medicines are the indispensable drugs that satisfy the priority health care needs of the population. Meanwhile, they are selected with due regard to rational use and equitable access on their efficacy, safety and comparative cost-effectiveness ${ }^{[6]}$. To better meet the necessary treatment needs, the Chinese government attaches great importance to the development and implementation of the Essential Medicine List (EML) ${ }^{[7]}$. In 2018, China re-adjusted the national EML, and increased the growth rate of oncology drugs by $35 \%$. In terms of cancer treatment, the 2018 edition of the "National Essential Medicine List" added 5 kinds of non-targeted drugs, including Ifosfamide, Gemcitabine, Pingyangmycin, Capecitabine, and Letrozole ${ }^{[8]}$. The supplementary medicines can greatly alleviate the burden of cancer treatment. After the implementation of the national essential medicine system, the production cost of essential medicines and unreasonable profit margins can be well controlled.

Recently, most of domestic studies on essential medicines focused on antibacterial drugs and medications for chronic diseases ${ }^{[8-10]}$. Their study can reflect the common problems in the availability and affordability of essential drugs in China, however, the question regarding whether it is the same case for anticancer still awaits further investigation. Therefore, we investigated the price, availability and affordability of anti-cancer drugs in 28 public hospitals in Hubei Province, in order to explore the factors influencing the shortage of medicines and rapidly increasing health expenditure, and accordingly, propose corresponding strategies to the government and pharmaceutical management institutes.

\section{Methods}

A cross sectional survey was conducted from November 2018 to January 2019. Data were collected on medicine price, availability and affordability information from tertiary hospitals and secondary hospitals in six cities of Hubei Province following WHO/HAl standardized approach and Health Action International (HAl) ${ }^{[11]}$.

\section{Study design/Sampling}

\section{Area selection}

We selected six cities as survey regions in Hubei Province by referring to the Gross Domestic Product (GDP) in 2018, according to which, we selected three cities in high-income (Wuhan, Yichang, Xiangyang) and low-income (Huanggang, Xianning, Suizhou) areas, respectively ${ }^{[12]}$. Hospitals were selected using a multistage clustered approach. Firstly, we chose at least one tertiary hospital as the survey anchor in each city (for instance, Tongji affiliated hospital of Tongji medical college was selected in Wuhan city).

Secondly, 2 or 3 secondary hospitals were randomly selected within a 3 hours' drive distance around the center survey anchor. A total of 13 tertiary hospitals and 15 secondary hospitals in the public sector were 
selected from six cities in Hubei Province. As private hospitals and retail pharmacies do not have the right to prescribe most oncology drugs, they were not included in this survey.

\section{Medicine selection}

A total of 32 medicines were included in this survey, referring to 2017 WHO Essential Medicine List (WEML) ${ }^{[13]}$ and 2018 National Essential Medicine List (NEML). Considering the common acute and medication conditions in tertiary and secondary hospitals of Hubei Province, we divided the selected medicines into two parts according to the available data collected from the sample hospitals. Firstly, the intersection area of anti-cancer drugs were from the WHO Essential Medicine List and National Essential Medicine List, which included 23 core essential medicines. Then, additional 9 sample drugs that were commonly used were also included in this survey list as supplementary according to oncologist's recommendation and the actual needs of local hospitals. For each medicine in the survey, data were collected for two medicine types: the originator brand (OBs), and the lowest-priced generic (LPGs) equivalent found at each hospital.

\section{Data collection}

With the aid of skilled pharmacy present, trained investigators visited the pharmacy department of the sampled tertiary and secondary hospitals. They collected information regarding the cost and availability using a standardized data collection form. At the end of each day, 2 students would enter the data into a designed MS Excel Workbook provided as a part of the WHO/ HAI methodology $[14,15]$. The items included in the standardized form were as follows: basic information of the facility (hospital name, hospital level, survey date), information of the medicine (medicine in stock in the hospital on the day of data collection, yes or no), dosage, strength, medicine type (OB/LPG), and the retail price at the time of survey (Table 1).

\section{Assessment of availability}

The availability of anticancer medicines was reported as the percentage (\%) of medicine outlets in which the information was documented by surveyed facilities on the day of data collection. Availability was classified as 4 degrees; Absent: 0 of facilities, suggested that we could not find these medicines in the facility; Low: $<50 \%$, these medicines were rarely purchased; Fairly high: $50 \%-80 \%$, these medicines were found in several facilities; High: $>80 \%$, most institutions sold these medicines. In addition, mean percentage availability of OBs and LPGs were calculated for the investigations ${ }^{[1]}$.

\section{Price assessment}

In order to make standard comparisons, price was evaluated by median price ratio (MPR) according to WHO/HAl approach, which is the ratio of median price of individual medicine obtained during the survey (1). Moreover, we used Management Sciences for Health (MSH2015) International Drug Price Indicator Guide ${ }^{[16]}$ as the source of reference prices. MPR was calculated using the formula given below: Median Price Ratio $(M P R)=$ Median local unit price / International reference unit price. It can provide a more 
intuitive data presentation for drug price monitoring: when the value of MPR is less than 1, it indicates that the price of the drug under investigation is lower than the international average standard. In other words, the price control is efficient. The value of MPR between 1 2 indicates that the drug price is acceptable; if MPR>2, this means that the drug price level is high and needs to be contained ${ }^{[17] .}$

\section{Affordability}

In general, the total cost of the unit treatment for chronic diseases with the standard dose of the drug is equivalent to 1 month's working days (daily wages) of the lowest paid non-technical government employee that enables him/her to purchase the standard course of cancer treatment. However, the duration of anti-cancer therapy may be longer. Moreover, the price of many anticancer drugs is too expensive, which causes heavy economic burden for cancer patients. Thus, with the aim to precisely assess the affordability, we used the approach proposed by Khatib and Sarwar $[18,19]$ : if the total expenditure of medicine in 30 days accounted for $20 \%$ or less of the minimum family monthly income, it was regarded as affordable ${ }^{[15]}$. The calculation formula was :

Affordability $=\frac{\text { Total costof drugsin } 30 \text { days }}{20 \% \text { of the minimum monthly household income }}$

Table 1 List of 32 anticancer medicines surveyed in the Hubei Province. 


\begin{tabular}{|c|c|c|c|c|c|}
\hline Name & Strength & Dosage Form & Volume & WHO EML & NEML \\
\hline Calcium Folinate & $15 \mathrm{mg}$ & VIAL & 1 & yes & yes \\
\hline Capecitabine & $500 \mathrm{mg}$ & TAB-CAP & 12 & yes & yes \\
\hline Carboplatin & 150 mg & VIAL & 1 & yes & yes \\
\hline Ciclosporin & $25 \mathrm{mg}$ & TAB-CAP & 50 & yes & yes \\
\hline Cisplatin & $10 \mathrm{mg}$ & VIAL & 1 & yes & yes \\
\hline Cyclophosphamide & $200 \mathrm{mg}$ & VIAL & 1 & yes & yes \\
\hline Cytarabine & 100 mg & VIAL & 1 & yes & yes \\
\hline Daunorubicin & $20 \mathrm{mg}$ & VIAL & 1 & yes & yes \\
\hline Docetaxel Trihydrate & $20 \mathrm{mg}$ & VIAL & 1 & yes & no \\
\hline Doxorubicin & $10 \mathrm{mg}$ & VIAL & 1 & yes & yes \\
\hline Etoposide & 20 mg/ml & VIAL & 1 & yes & yes \\
\hline Fluorouracil & $25 \mathrm{mg}$ & VIAL & 1 & yes & yes \\
\hline Gemcitabine & $200 \mathrm{mg}$ & VIAL & 1 & yes & yes \\
\hline Ifosfamide & $500 \mathrm{mg}$ & VIAL & 1 & yes & yes \\
\hline Imatinib & 100 mg & TAB-CAP & 60 & yes & yes \\
\hline Irinotecan & $20 \mathrm{mg} / \mathrm{ml}$ & VIAL & 1 & yes & no \\
\hline Mercaptopurine & $50 \mathrm{mg}$ & TAB-CAP & 50 & yes & yes \\
\hline Mesna & $100 \mathrm{mg} / \mathrm{ml}$ & AMPOULE & 1 & yes & yes \\
\hline Methotrexate Sodium & 10 mg/ml & VIAL & 1 & yes & yes \\
\hline Methylprednisolone & $500 \mathrm{mg}$ & VIAL & 1 & yes & yes \\
\hline Mycophenolate & $250 \mathrm{mg}$ & TAB-CAP & 40 & no & \\
\hline Mofetil & & & & & yes \\
\hline Oxaliplatin & $50 \mathrm{mg}$ & VIAL & 1 & yes & yes \\
\hline Paclitaxel & $6 \mathrm{mg} / \mathrm{ml}$ & VIAL & 1 & yes & yes \\
\hline Tamoxifen Citrate & $10 \mathrm{mg}$ & TAB-CAP & 60 & yes & yes \\
\hline
\end{tabular}




\begin{tabular}{|llllll|}
\hline Name & Strength & Dosage Form & Volume & WHO EML & NEML \\
\hline Vincristine & $1 \mathrm{mg}$ & VIAL & 1 & yes & yes \\
\hline Letrozole & $2.5 \mathrm{mg}$ & TAB-CAP & 10 & no & yes \\
\hline Gefitinib & $25 \mathrm{mg}$ & TAB-CAP & 10 & no & yes \\
\hline Bicalutamide & $50 \mathrm{mg}$ & TAB-CAP & 28 & yes & no \\
\hline Hydroxycarbamide & $500 \mathrm{mg}$ & TAB-CAP & 100 & yes & yes \\
\hline Vinorelbine & $10 \mathrm{mg}$ & VIAL & 1 & yes & no \\
\hline Tacrolimus & $1 \mathrm{mg}$ & TAB-CAP & 10 & no & no \\
\hline Ondansetron & $4 \mathrm{mg}$ & TAB-CAP & 10 & yes & yes \\
\hline
\end{tabular}

\section{Results}

We calculated the availability of originator brands (OBs) and lowest priced generics (LPGs) across the 32 anti-cancer medicines. The mean availability of surveyed medicines in both tertiary and secondary hospitals was $40.88 \%$ for LPGs, and $7.75 \%$ for OBs. In tertiary hospitals, the average availability of OBs was $13.70 \%$ and that of the LPGs was $38.84 \%$. In contrast, the mean availability of OBs was $6.67 \%$, and LPGs accounted for $42.92 \%$ in secondary hospitals (Table 2 ).

In this survey, $10 \mathrm{OBs}$ of 32 medicines were not available in tertiary hospitals nor secondary hospitals. The top three percentage availability of OBs in tertiary hospitals included Bicalutamide (47.62\%), Methylprednisolone (38.10\%) and Gefitinib (28.57\%). For LPGs, the availability of nine medicines was over $50 \%$. Besides, Oxaliplatin and Gemcitabine were maximal for $61.90 \%$ in tertiary hospitals (Fig1). Two medicines with the highest availability in the secondary hospitals were Cyclophosphamide (93.33\%) and Oxaliplatin (93.33\%). A total of $21 \mathrm{OBs}$ were not available during this investigation. Nevertheless, most LPGs were available in secondary hospitals (Fig2). In general, the availability of the selected medicines in tertiary hospitals was higher than that in secondary hospitals.

Table 2 Availability of anti-cancer medicines in tertiary and Secondary hospitals 


\begin{tabular}{|c|c|c|c|c|c|c|}
\hline \multirow[t]{2}{*}{ Medicine name } & \multicolumn{2}{|l|}{$\mathrm{OBs}(\%)$} & \multicolumn{2}{|l|}{ LPGs(\%) } & \multicolumn{2}{|c|}{ Total(\%) } \\
\hline & $\begin{array}{l}\text { Tertiary } \\
\text { hospital }\end{array}$ & $\begin{array}{l}\text { Secondary } \\
\text { hospital }\end{array}$ & $\begin{array}{l}\text { Tertiary } \\
\text { hospital }\end{array}$ & $\begin{array}{l}\text { Secondary } \\
\text { hospital }\end{array}$ & OBs & LPGs \\
\hline Calcium Folinate & 0.00 & 0.00 & 47.62 & 60.0 & 0.00 & 53.81 \\
\hline Capecitabine & 23.81 & 40.00 & 52.38 & 66.67 & 31.90 & 59.52 \\
\hline Carboplatin & 0.00 & 0.00 & 52.38 & 33.33 & 0.00 & 42.86 \\
\hline Ciclosporin & 23.08 & 6.67 & 38.10 & 13.33 & 10.48 & 25.71 \\
\hline Cisplatin & 0.00 & 0.00 & 42.86 & 53.33 & 0.00 & 48.10 \\
\hline Cyclophosphamide & 15.38 & 0.00 & 52.38 & 93.33 & 4.76 & 72.86 \\
\hline Cytarabine & 7.69 & 0.00 & 28.57 & 13.33 & 2.38 & 20.95 \\
\hline Daunorubicin & 0.00 & 0.00 & 28.57 & 26.67 & 0.00 & 27.62 \\
\hline $\begin{array}{l}\text { Docetaxel } \\
\text { Trihydrate }\end{array}$ & 7.69 & 0.00 & 47.62 & 73.33 & 2.38 & 60.48 \\
\hline Doxorubicin & 0.00 & 0.00 & 23.81 & 33.33 & 0.00 & 28.57 \\
\hline Etoposide & 0.00 & 0.00 & 57.14 & 80.00 & 0.00 & 68.57 \\
\hline Fluorouracil & 7.69 & 0.00 & 33.33 & 46.67 & 2.38 & 40.00 \\
\hline Gemcitabine & 7.69 & 0.00 & 61.90 & 73.33 & 2.38 & 67.62 \\
\hline Ifosfamide & 23.08 & 0.00 & 33.33 & 13.33 & 7.14 & 23.33 \\
\hline Imatinib & 7.69 & 6.67 & 38.10 & 20.00 & 5.71 & 29.05 \\
\hline Irinotecan & 7.69 & 6.67 & 47.62 & 46.67 & 5.71 & 47.14 \\
\hline Mercaptopurine & 0.00 & 0.00 & 4.76 & 0.00 & 0.00 & 2.38 \\
\hline Mesna & 7.69 & 0.00 & 38.10 & 20.00 & 2.38 & 29.05 \\
\hline $\begin{array}{l}\text { Methotrexate } \\
\text { Sodium }\end{array}$ & 0.00 & 0.00 & 52.38 & 66.67 & 0.00 & 59.52 \\
\hline Methylprednisolone & 38.10 & 40.00 & 9.52 & 26.67 & 39.05 & 18.10 \\
\hline $\begin{array}{l}\text { Mycophenolate } \\
\text { Mofetil }\end{array}$ & 7.69 & 13.33 & 28.57 & 13.33 & 9.05 & 20.95 \\
\hline Oxaliplatin & 15.38 & 6.67 & 61.90 & 93.33 & 8.10 & 77.62 \\
\hline Paclitaxel & 23.08 & 0.00 & 42.86 & 40.00 & 7.14 & 41.43 \\
\hline Tamoxifen Citrate & 0.00 & 0.00 & 52.38 & 80.00 & 0.00 & 66.19 \\
\hline Vincristine & 0.00 & 6.67 & 28.57 & 46.67 & 3.33 & 37.62 \\
\hline
\end{tabular}




\begin{tabular}{|lllllll|}
\hline Medicine name & OBs(\%) & \multicolumn{3}{l}{ LPGs(\%) } & \multicolumn{3}{l|}{ Total(\%) } \\
\hline & $\begin{array}{l}\text { Tertiary } \\
\text { hospital }\end{array}$ & $\begin{array}{l}\text { Secondary } \\
\text { hospital }\end{array}$ & $\begin{array}{l}\text { Tertiary } \\
\text { hospital }\end{array}$ & $\begin{array}{l}\text { Secondary } \\
\text { hospital }\end{array}$ & OBs & LPGs \\
\hline Letrozole & 23.08 & 20.00 & 52.38 & 66.67 & 17.14 & 59.52 \\
\hline Gefitinib & 28.57 & 13.33 & 28.57 & 20.00 & 23.33 & 24.29 \\
\hline Bicalutamide & 47.62 & 40.00 & 23.81 & 20.00 & 43.81 & 21.90 \\
\hline Hydroxycarbamide & 0.00 & 0.00 & 38.10 & 40.00 & 0.00 & 39.05 \\
\hline Vinorelbine & 7.69 & 0.00 & 42.86 & 53.33 & 2.38 & 48.10 \\
\hline Tacrolimus & 15.38 & 13.33 & 28.57 & 20.00 & 11.43 & 24.29 \\
\hline Ondansetron & 0.00 & 0.00 & 23.81 & 20.00 & 0.00 & 21.90 \\
\hline Mean Percent & 13.70 & 6.67 & 38.84 & 42.92 & 7.57 & 40.88 \\
\hline
\end{tabular}

Table 3 illustrates the median price patients paid and MPR in tertiary and secondary hospitals for 32 anticancer medicines in Hubei Province. The results showed that the price of all medicines varied from $¥ 0.49$ to $¥ 2290.12$, and the median patient prices of OB for Oxaliplatin ( $¥ 2290.12$ ) ranked first. Especially,

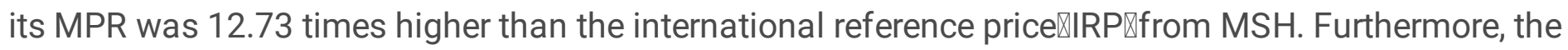
MPR of 3 OBs were more than twice that of IRP in tertiary hospital: Cytarabine (MPR=17.15), Ifosfamide $(M P R=2.30)$, Paclitaxel (MPR=2.81), respectively. The MPR value of sample medicines in secondary hospitals were less than twice, except Letrozole (MPR=3.26).

Table 3 The median patient price and MPR of anticancer medicines in tertiary and secondary hospitals in Hubei Province. 


\begin{tabular}{|c|c|c|c|c|c|}
\hline \multirow[t]{2}{*}{ Medicine name } & \multirow[t]{2}{*}{ Medicine type } & \multicolumn{2}{|c|}{ Tertiary hospital[n=13] } & \multicolumn{2}{|c|}{$\begin{array}{l}\text { Secondary hospital } \\
(n=15)\end{array}$} \\
\hline & & Median Price & MPR & Median Price & MPR \\
\hline \multirow[t]{2}{*}{ Calcium Folinate } & $\mathrm{OB}$ & NA & NA & NA & NA \\
\hline & LPG & 16.18 & 1.00 & 16.18 & 1.00 \\
\hline \multirow[t]{2}{*}{ Capecitabine } & $\mathrm{OB}$ & 30.72 & 1.00 & 30.72 & 1.00 \\
\hline & LPG & 8.16 & 0.27 & 11.14 & 0.36 \\
\hline \multirow[t]{2}{*}{ Carboplatin } & $\mathrm{OB}$ & NA & NA & NA & NA \\
\hline & LPG & 53.90 & 1.00 & 53.90 & 1.00 \\
\hline \multirow[t]{2}{*}{ Ciclosporin } & $\mathrm{OB}$ & 9.15 & 1.00 & NA & NA \\
\hline & LPG & 5.18 & 0.57 & 5.36 & 0.59 \\
\hline \multirow[t]{2}{*}{ Cisplatin } & $\mathrm{OB}$ & NA & NA & NA & NA \\
\hline & LPG & 7.57 & 1.04 & 7.30 & 1.00 \\
\hline \multirow[t]{2}{*}{ Cyclophosphamide } & $\mathrm{OB}$ & NA & NA & NA & NA \\
\hline & LPG & 25.20 & 1.00 & 25.20 & 1.00 \\
\hline \multirow[t]{2}{*}{ Cytarabine } & $\mathrm{OB}$ & 140.49 & 17.15 & NA & NA \\
\hline & LPG & 8.19 & 1.00 & 8.09 & 0.99 \\
\hline \multirow[t]{2}{*}{ Daunorubicin } & $\mathrm{OB}$ & NA & NA & NA & NA \\
\hline & LPG & 27.15 & 1.00 & 27.15 & 1.00 \\
\hline \multirow[t]{2}{*}{ Docetaxel } & $\mathrm{OB}$ & NA & NA & NA & NA \\
\hline & LPG & 204.84 & 0.66 & 123.00 & 0.40 \\
\hline \multirow[t]{2}{*}{ Doxorubicin } & $\mathrm{OB}$ & NA & NA & NA & NA \\
\hline & LPG & 23.28 & 1.00 & 23.28 & 1.00 \\
\hline \multirow[t]{2}{*}{ Etoposide } & $\mathrm{OB}$ & NA & NA & NA & NA \\
\hline & LPG & 7.79 & 0.78 & 9.28 & 0.93 \\
\hline \multirow[t]{2}{*}{ Fluorouracil } & $\mathrm{OB}$ & NA & NA & NA & NA \\
\hline & LPG & 9.80 & 1.98 & 5.10 & 1.03 \\
\hline \multirow[t]{2}{*}{ Gemcitabine } & $\mathrm{OB}$ & NA & NA & NA & NA \\
\hline & LPG & 150.99 & 1.00 & 79.75 & 0.53 \\
\hline
\end{tabular}




\begin{tabular}{|c|c|c|c|c|c|}
\hline \multirow[t]{2}{*}{ Medicine name } & \multirow[t]{2}{*}{ Medicine type } & \multicolumn{2}{|c|}{ Tertiary hospital[n=13ם } & \multicolumn{2}{|c|}{$\begin{array}{l}\text { Secondary hospital } \\
(n=15)\end{array}$} \\
\hline & & Median Price & MPR & Median Price & MPR \\
\hline \multirow[t]{2}{*}{ Ifosfamide } & $\mathrm{OB}$ & 107.88 & 2.30 & NA & NA \\
\hline & LPG & 47.00 & 1.00 & 42.21 & 0.90 \\
\hline \multirow[t]{2}{*}{ Imatinib } & $\mathrm{OB}$ & NA & NA & NA & NA \\
\hline & LPG & 14.05 & 0.70 & 14.05 & 0.70 \\
\hline \multirow[t]{2}{*}{ Irinotecan } & $\mathrm{OB}$ & 385.87 & 1.66 & NA & NA \\
\hline & LPG & 252.44 & 1.08 & 252.44 & 1.08 \\
\hline \multirow[t]{2}{*}{ Mercaptopurine } & $\mathrm{OB}$ & NA & NA & NA & NA \\
\hline & LPG & 0.62 & 0.26 & NA & NA \\
\hline \multirow[t]{2}{*}{ Mesna } & $\mathrm{OB}$ & NA & NA & NA & NA \\
\hline & LPG & 8.80 & 1.00 & 8.80 & 1.00 \\
\hline \multirow[t]{2}{*}{ Methotrexate Sodium } & $\mathrm{OB}$ & NA & NA & NA & NA \\
\hline & LPG & 19.60 & 1.00 & 19.60 & 1.16 \\
\hline Methylprednisolone & $\mathrm{OB}$ & 123.96 & 1.00 & 125.83 & 1.02 \\
\hline \multirow[t]{2}{*}{ Mycophenolate Mofetil } & $\mathrm{OB}$ & 14.85 & 1.85 & 14.87 & 1.85 \\
\hline & LPG & 8.04 & 1.00 & 8.31 & 1.03 \\
\hline \multirow[t]{2}{*}{ Oxaliplatin } & $\mathrm{OB}$ & 2290.12 & 12.73 & NA & NA \\
\hline & LPG & 179.88 & 1.00 & 73.10 & 0.41 \\
\hline \multirow[t]{2}{*}{ Paclitaxel } & $\mathrm{OB}$ & 94.19 & 2.81 & NA & NA \\
\hline & LPG & 33.55 & 1.00 & 15.99 & 0.48 \\
\hline \multirow[t]{2}{*}{ Tamoxifen Citrate } & $\mathrm{OB}$ & NA & 1.09 & NA & NA \\
\hline & LPG & 0.58 & NA & 0.56 & 1.06 \\
\hline \multirow[t]{2}{*}{ Vincristine } & $\mathrm{OB}$ & NA & NA & NA & NA \\
\hline & LPG & 43.47 & 3.25 & 9.49 & 1.00 \\
\hline \multirow[t]{2}{*}{ Letrozole } & $\mathrm{OB}$ & 37.55 & 1.00 & 37.73 & 3.26 \\
\hline & LPG & 10.51 & 1.00 & 11.56 & 1.00 \\
\hline Gefitinib & $\mathrm{OB}$ & 228 & 0.69 & 231.90 & 1.02 \\
\hline
\end{tabular}




\begin{tabular}{|c|c|c|c|c|c|}
\hline \multirow[t]{2}{*}{ Medicine name } & \multirow[t]{2}{*}{ Medicine type } & \multicolumn{2}{|c|}{ Tertiary hospital[n=13ם } & \multicolumn{2}{|c|}{$\begin{array}{l}\text { Secondary hospital } \\
(n=15)\end{array}$} \\
\hline & & Median Price & MPR & Median Price & MPR \\
\hline & LPG & 158.4 & 1.32 & 158.50 & 0.70 \\
\hline \multirow[t]{2}{*}{ Bicalutamide } & $\mathrm{OB}$ & 40.83 & 0.90 & 40.83 & 1.32 \\
\hline & LPG & 27.88 & NA & 40.83 & 1.32 \\
\hline \multirow[t]{2}{*}{ Hydroxycarbamide } & $\mathrm{OB}$ & NA & 1.00 & NA & NA \\
\hline & LPG & 0.49 & NA & 0.49 & 1.00 \\
\hline \multirow[t]{2}{*}{ Vinorelbine } & $\mathrm{OB}$ & NA & NA & NA & NA \\
\hline & LPG & 134.82 & 1.00 & 134.82 & 1.00 \\
\hline \multirow[t]{2}{*}{ Tacrolimus } & $\mathrm{OB}$ & 23.11 & 1.98 & 23.21 & 1.99 \\
\hline & LPG & 10.51 & 0.90 & 16.32 & 1.40 \\
\hline \multirow[t]{2}{*}{ Ondansetron } & $\mathrm{OB}$ & NA & NA & NA & NA \\
\hline & LPG & 13.89 & 1.16 & 22.24 & 1.86 \\
\hline
\end{tabular}

In order to compare the difference of drug availability and MPR value between secondary and tertiary hospitals, we made 4 scatter plots of the availability and MPR value of 32 anticancer drugs (Figure 3 ). In tertiary hospitals, for OBs, two points located in the upper left area of the graph, which were cytarabine and oxaliplatin, whose MPR was more than 10 times. However, their availability was quite low. The medicines with higher availability of OBs were Gefitinib, Bicalutamide and Methylprednisolone with low MPR. For LPGs, Vincristine had the highest MPR value (3.25), and its availability was low. Nine medicines had fairly high availability with acceptable MPR value. In secondary hospitals, for OBs, only 7 drugs with MPR value could be obtained. Figure 3 indicates Letrozole with high availability and MPR. For LPGs, 11 medicines were highly available. Moreover, the MPR of all all medicines were within the acceptable range.

Table 4 shows the affordability of the anticancer medicines based on the duration of treatment. Overall, the mean affordability of the studied medicines was 2.89 , and the LPGs was better than that of OBs. For 31 LPGs, the mean affordability value was 1.79, which was within normal range. However, for 14 OBs, the mean value of affordability was 5.29. Further, irinotecan (13.89) and oxaliplatin (11.91) induced large payment burden, as the cost of 30 days dosage was higher than 10 times of $20 \%$ household minimum monthly income. In the surveyed hospitals, the affordability of $50 \%$ of the OBs exceeded the mean affordability value (5.29), and $22.58 \%$ of LPGs was over the average affordability.

Table 4 Affordability of anticancer medicines based on $20 \%$ of the minimum household monthly income in Hubei Province. 


\begin{tabular}{|c|c|c|c|c|c|c|}
\hline Medicine name & $\begin{array}{l}\text { Medicine } \\
\text { type }\end{array}$ & Dosage & strength & $\begin{array}{l}\text { Total course of } \\
\text { treatment }\end{array}$ & $\begin{array}{l}\text { Median } \\
\text { patient } \\
\text { price }\end{array}$ & $\begin{array}{l}\text { Afford- } \\
\text { ability }\end{array}$ \\
\hline \multirow[t]{2}{*}{ Calcium Folinate } & $\mathrm{OB}$ & VIAL & $15 \mathrm{mg}$ & NA & NA & 0.00 \\
\hline & LPG & VIAL & $50 \mathrm{mg}$ & 10 & 16.18 & 0.32 \\
\hline \multirow[t]{2}{*}{ Capecitabine } & $\mathrm{OB}$ & $\begin{array}{l}\text { TAB- } \\
\text { CAP }\end{array}$ & $500 \mathrm{mg}$ & 105 & 30.72 & 6.45 \\
\hline & LPG & $\begin{array}{l}\text { TAB- } \\
\text { CAP }\end{array}$ & $500 \mathrm{mg}$ & 105 & 8.16 & 1.71 \\
\hline \multirow[t]{2}{*}{ Carboplatin } & $\mathrm{OB}$ & VIAL & $150 \mathrm{mg}$ & 6 & NA & 0.00 \\
\hline & LPG & VIAL & $150 \mathrm{mg}$ & 6 & 53.90 & 0.65 \\
\hline \multirow[t]{2}{*}{ Ciclosporin } & $\mathrm{OB}$ & $\begin{array}{l}\text { TAB- } \\
\text { CAP }\end{array}$ & $25 \mathrm{mg}$ & 30 & 9.15 & 0.55 \\
\hline & LPG & $\begin{array}{l}\text { TAB- } \\
\text { CAP }\end{array}$ & $25 \mathrm{mg}$ & 30 & 5.18 & 0.31 \\
\hline \multirow[t]{2}{*}{ Cisplatin } & $\mathrm{OB}$ & VIAL & $10 \mathrm{mg}$ & 10 & NA & 0.00 \\
\hline & LPG & VIAL & $10 \mathrm{mg}$ & 10 & 7.57 & 0.15 \\
\hline \multirow[t]{2}{*}{ Cyclophosphamide } & $\mathrm{OB}$ & VIAL & $200 \mathrm{mg}$ & 20 & NA & 0.00 \\
\hline & LPG & VIAL & $200 \mathrm{mg}$ & 20 & 25.20 & 1.01 \\
\hline \multirow[t]{2}{*}{ Cytarabine } & $\mathrm{OB}$ & VIAL & $100 \mathrm{mg}$ & 20 & 140.49 & 5.62 \\
\hline & LPG & VIAL & $100 \mathrm{mg}$ & 20 & 8.19 & 0.33 \\
\hline \multirow[t]{2}{*}{ Daunorubicin } & $\mathrm{OB}$ & VIAL & $20 \mathrm{mg}$ & 6 & NA & 0.00 \\
\hline & LPG & VIAL & $20 \mathrm{mg}$ & 6 & 27.15 & 0.33 \\
\hline \multirow[t]{2}{*}{ Docetaxel } & $\mathrm{OB}$ & VIAL & $20 \mathrm{mg}$ & 3.75 & NA & 0.00 \\
\hline & LPG & VIAL & $20 \mathrm{mg}$ & 3.75 & 204.84 & 1.54 \\
\hline \multirow[t]{2}{*}{ Doxorubicin } & $\mathrm{OB}$ & VIAL & $10 \mathrm{mg}$ & 6 & NA & 0.00 \\
\hline & LPG & VIAL & $10 \mathrm{mg}$ & 6 & 23.28 & 0.28 \\
\hline \multirow[t]{2}{*}{ Etoposide } & $\mathrm{OB}$ & VIAL & $\begin{array}{l}20 \\
\mathrm{mg} / \mathrm{ml}\end{array}$ & 30 & NA & 0.00 \\
\hline & LPG & VIAL & $\begin{array}{l}20 \\
\mathrm{mg} / \mathrm{ml}\end{array}$ & 30 & 7.79 & 0.47 \\
\hline \multirow[t]{2}{*}{ Fluorouracil } & $\mathrm{OB}$ & VIAL & $25 \mathrm{mg}$ & 300 & NA & 0.00 \\
\hline & LPG & VIAL & $25 \mathrm{mg}$ & 300 & 9.80 & 5.88 \\
\hline
\end{tabular}




\begin{tabular}{|c|c|c|c|c|c|c|}
\hline Medicine name & $\begin{array}{l}\text { Medicine } \\
\text { type }\end{array}$ & Dosage & strength & $\begin{array}{l}\text { Total course of } \\
\text { treatment }\end{array}$ & $\begin{array}{l}\text { Median } \\
\text { patient } \\
\text { price }\end{array}$ & $\begin{array}{l}\text { Afford- } \\
\text { ability }\end{array}$ \\
\hline \multirow[t]{2}{*}{ Gemcitabine } & $\mathrm{OB}$ & VIAL & $200 \mathrm{mg}$ & 15 & NA & 0.00 \\
\hline & LPG & VIAL & $200 \mathrm{mg}$ & 15 & 150.99 & 4.53 \\
\hline \multirow[t]{2}{*}{ Ifosfamide } & $\mathrm{OB}$ & VIAL & $500 \mathrm{mg}$ & 15 & 107.88 & 3.24 \\
\hline & LPG & VIAL & $500 \mathrm{mg}$ & 15 & 47.00 & 1.41 \\
\hline \multirow[t]{2}{*}{ Imatinib } & $\mathrm{OB}$ & $\begin{array}{l}\text { TAB- } \\
\text { CAP }\end{array}$ & $100 \mathrm{mg}$ & 112 & NA & 0.00 \\
\hline & LPG & $\begin{array}{l}\text { TAB- } \\
\text { CAP }\end{array}$ & $100 \mathrm{mg}$ & 112 & 14.05 & 3.15 \\
\hline \multirow[t]{2}{*}{ Irinotecan } & $\mathrm{OB}$ & $\begin{array}{l}\text { TAB- } \\
\text { CAP }\end{array}$ & $\begin{array}{l}20 \\
\mathrm{mg} / \mathrm{ml}\end{array}$ & 18 & 385.87 & 13.89 \\
\hline & LPG & $\begin{array}{l}\text { TAB- } \\
\text { CAP }\end{array}$ & $\begin{array}{l}20 \\
\mathrm{mg} / \mathrm{ml}\end{array}$ & 18 & 252.44 & 9.09 \\
\hline \multirow[t]{2}{*}{ Mercaptopurine } & $\mathrm{OB}$ & $\begin{array}{l}\text { TAB- } \\
\text { CAP }\end{array}$ & $50 \mathrm{mg}$ & 240 & NA & 0.00 \\
\hline & LPG & $\begin{array}{l}\text { TAB- } \\
\text { CAP }\end{array}$ & $50 \mathrm{mg}$ & 240 & 0.62 & 0.30 \\
\hline \multirow[t]{2}{*}{ Mesna } & $\mathrm{OB}$ & VIAL & $\begin{array}{l}100 \\
\mathrm{mg} / \mathrm{ml}\end{array}$ & 10 & NA & 0.00 \\
\hline & LPG & VIAL & $\begin{array}{l}100 \\
\mathrm{mg} / \mathrm{ml}\end{array}$ & 10 & 8.80 & 0.18 \\
\hline \multirow{2}{*}{$\begin{array}{l}\text { Methotrexate } \\
\text { Sodium }\end{array}$} & $\mathrm{OB}$ & VIAL & $10 \mathrm{mg} / \mathrm{ml}$ & 32 & NA & 0.00 \\
\hline & LPG & VIAL & $10 \mathrm{mg} / \mathrm{ml}$ & 32 & 9.80 & 0.63 \\
\hline \multirow[t]{2}{*}{ Methylprednisolone } & $\mathrm{OB}$ & VIAL & $500 \mathrm{mg}$ & 20 & 123.96 & 4.96 \\
\hline & LPG & VIAL & $500 \mathrm{mg}$ & 20 & 161.72 & 6.47 \\
\hline \multirow[t]{2}{*}{$\begin{array}{l}\text { Mycophenolate } \\
\text { Mofetil }\end{array}$} & $\mathrm{OB}$ & $\begin{array}{l}\text { TAB- } \\
\text { CAP }\end{array}$ & $250 \mathrm{mg}$ & 240 & 14.85 & 7.13 \\
\hline & LPG & $\begin{array}{l}\text { TAB- } \\
\text { CAP }\end{array}$ & $250 \mathrm{mg}$ & 240 & 8.04 & 3.86 \\
\hline \multirow[t]{2}{*}{ Oxaliplatin } & $\mathrm{OB}$ & VIAL & $50 \mathrm{mg}$ & 2.6 & 2290.12 & 11.91 \\
\hline & LPG & VIAL & $50 \mathrm{mg}$ & 2.6 & 179.88 & 0.94 \\
\hline \multirow[t]{2}{*}{ Paclitaxel } & $\mathrm{OB}$ & VIAL & $6 \mathrm{mg} / \mathrm{ml}$ & 30 & 94.19 & 5.65 \\
\hline & LPG & VIAL & 6 mg/ml & 30 & 33.55 & 2.01 \\
\hline
\end{tabular}




\begin{tabular}{|c|c|c|c|c|c|c|}
\hline Medicine name & $\begin{array}{l}\text { Medicine } \\
\text { type }\end{array}$ & Dosage & strength & $\begin{array}{l}\text { Total course of } \\
\text { treatment }\end{array}$ & $\begin{array}{l}\text { Median } \\
\text { patient } \\
\text { price }\end{array}$ & $\begin{array}{l}\text { Afford- } \\
\text { ability }\end{array}$ \\
\hline \multirow[t]{2}{*}{ Tamoxifen Citrate } & $\mathrm{OB}$ & & $10 \mathrm{mg}$ & 30 & NA & 0.00 \\
\hline & LPG & $\begin{array}{l}\text { TAB- } \\
\text { CAP }\end{array}$ & $10 \mathrm{mg}$ & 30 & 0.58 & 0.03 \\
\hline \multirow[t]{2}{*}{ Vincristine } & $\mathrm{OB}$ & VIAL & $1 \mathrm{mg}$ & 4 & NA & 0.00 \\
\hline & LPG & VIAL & $1 \mathrm{mg}$ & 4 & 134.82 & 1.08 \\
\hline \multirow[t]{2}{*}{ Letrozole } & $\mathrm{OB}$ & $\begin{array}{l}\text { TAB- } \\
\text { CAP }\end{array}$ & $2.5 \mathrm{mg}$ & 75 & 23.11 & 3.47 \\
\hline & LPG & $\begin{array}{l}\text { TAB- } \\
\text { CAP }\end{array}$ & $2.5 \mathrm{mg}$ & 75 & 10.51 & 1.58 \\
\hline \multirow[t]{2}{*}{ Gefitinib } & $\mathrm{OB}$ & $\begin{array}{l}\text { TAB- } \\
\text { CAP }\end{array}$ & $25 \mathrm{mg}$ & 30 & NA & 0.00 \\
\hline & LPG & $\begin{array}{l}\text { TAB- } \\
\text { CAP }\end{array}$ & $25 \mathrm{mg}$ & 30 & 13.89 & 0.83 \\
\hline \multirow[t]{2}{*}{ Bicalutamide } & $\mathrm{OB}$ & $\begin{array}{l}\text { TAB- } \\
\text { CAP }\end{array}$ & $50 \mathrm{mg}$ & 30 & 134.82 & 8.09 \\
\hline & LPG & $\begin{array}{l}\text { TAB- } \\
\text { CAP }\end{array}$ & $50 \mathrm{mg}$ & 7 & 23.11 & 0.32 \\
\hline \multirow[t]{2}{*}{ Hydroxycarbamide } & $\mathrm{OB}$ & $\begin{array}{l}\text { TAB- } \\
\text { CAP }\end{array}$ & $500 \mathrm{mg}$ & 7 & 10.51 & 0.15 \\
\hline & LPG & $\begin{array}{l}\text { TAB- } \\
\text { CAP }\end{array}$ & $500 \mathrm{mg}$ & 7 & NA & 0.00 \\
\hline \multirow[t]{2}{*}{ Vinorelbine } & $\mathrm{OB}$ & VIAL & $10 \mathrm{mg}$ & 7 & 13.89 & 0.19 \\
\hline & LPG & VIAL & $10 \mathrm{mg}$ & 7 & 134.82 & 1.89 \\
\hline \multirow[t]{2}{*}{ Tacrolimus } & $\mathrm{OB}$ & $\begin{array}{l}\text { TAB- } \\
\text { CAP }\end{array}$ & $1 \mathrm{mg}$ & 60 & 23.11 & 2.77 \\
\hline & LPG & $\begin{array}{l}\text { TAB- } \\
\text { CAP }\end{array}$ & $1 \mathrm{mg}$ & 6 & 10.51 & 0.13 \\
\hline \multirow[t]{2}{*}{ Ondansetron } & $\mathrm{OB}$ & $\begin{array}{l}\text { TAB- } \\
\text { CAP }\end{array}$ & $4 \mathrm{mg}$ & 80 & NA & 0.00 \\
\hline & LPG & $\begin{array}{l}\text { TAB- } \\
\text { CAP }\end{array}$ & $4 \mathrm{mg}$ & 80 & 13.89 & 2.22 \\
\hline Mean affordability & OB & 5.29 & LPG & 1.79 & Total & 2.89 \\
\hline
\end{tabular}

*Total course of treatment is calculated as 30 days' dose of anticancer medicines 
Figure 4 shows that the monthly medicine expenditure of 3 OBs (Irinotecan, Bicalutamide, Oxaliplatin) exceeded 4000 RMB, and the cost of five medicines (Paclitaxel, Methylprednisolone, Mycophenolate Mofetil, Cytarabine and Capecitabine) were within 2000-4000 RMB. For LPGs, the 30 day treatment expenditure of four medicines (Irinotecan, Methylprednisolone, Gemcitabine, Fluorouracil) were over 2000 $\mathrm{RMB}$, and the cost of most of the rest medicines were around $1000 \mathrm{RMB}$.

\section{Discussion}

\section{Availability of surveyed medicines}

The study evaluated the availability, price and affordability of 32 anticancer medicines in Hubei Province by using the WHO and HAl standard methodology. After investigating 13 tertiary hospitals and 15 secondary hospitals, we found that nine LPGs out of the surveyed medicines met the fairly high level of availability in tertiary hospitals, and four LPGs were in the high level availability in the secondary hospitals. Overall, the average availability of OB medicines was lower than that of LPG medicines (Figure1-2). The findings are consistent with prior surveys in developing countries ${ }^{[14,20,21]}$. They found that most of the $\mathrm{OB}$ medicines were produced by large international manufacturers. Due to the lack of effective external supervision and approval for the OB medicines, price leading and brand premiums led to the confusingly higher price.

Compared with secondary hospitals, the availability of surveyed medicines were higher in tertiary hospitals. Since radiotherapy and chemotherapy of tumor are very professional and expensive, they are mostly concentrated in large specialized hospitals or departments of tertiary hospitals, which contributed to the higher availability ${ }^{[22]}$.

In general, the price of the OBs was high because of its core patent right, as well as their high quality and stability were unique advantage ${ }^{[1,23]}$. However, LPG can be used as a competitive product to make up for its lack of accessibility ${ }^{[24]}$. Especially in low and middle-income countries, hospitals will take into account the actual purchasing power of patients, and appropriately reduce the inventory proportion of OBs in hospitals ${ }^{[17,25]}$. The findings of our study showed that the availability of some medicines was relatively high, including Gemcitabine, Etoposide, Oxaliplatin, Cyclophosphamide, Tamoxifen, etc. the underlying reason may be that these medicines have a comparatively low toxicity and high effectiveness

[26], and they are widely used in the treatment of some high incidence and mortality cancers, such as lung cancer, gastric cancer, colon cancer in China [27].

\section{Price comparison}

As presented in Figure 3, most of the LPGs for anticancer could be acquired with acceptable price. Meanwhile, for OBs, the MPR value of Cytarabine, Oxaliplatin and Paclitaxel were much higher than the international reference price from MSH. Compared with LPGs, the relatively retail prices for OBs were much higher. This may be explained by the large patient's rigid demand for the above three categories of 
medicines. In addition, the particularity of pharmaceutical production technical and core-patient barriers made it difficult for many domestic generic drugs to reach the ideal level and curative effect of the originator brand drugs ${ }^{[28]}$. Therefore, the mentioned factors above contributed to the high price of OBs in China.

The government has been very concerned about the field of medicine prices. In recent years, it has also issued relevant policies to curb the disordered competition in pharmaceutical market. Launching the new direction of "purchasing with quantity, linking quantity with price and combining bidding and purchasing" has pushed the price of national essential drugs back to a reasonable level ${ }^{[8,29]}$. Especially, after the conformity evaluation, generic drugs gradually occupy the share of originator brand drugs and expand the OBs' substitution in favour of lowering public health expenditure in hospitals ${ }^{[30]}$. Diversified and fair competitions between OBs and LPGs may bring more preferential benefits to patients.

\section{Affordability}

For the patients from low-income families in Hubei Province, they are still unable to afford course of treatment. Economic burden of disease becomes the major barrier that affects their access to effective targeted anticancer medicines. In this study, the measurement affordability based on the total cost of treatment and the estimation of household income. The top five medicines with poor affordability were: Irinotecan, Oxaliplatin, Bicalutamide and Mycophenolate Mofetil, Methylprednisolone, which were more than 6 times of the $20 \%$ minimal monthly household income. The heavy payment burden may be caused by different dosages of 30 day treatment course and the unit price of each medicine. Hence, the government should establish special funds to support low-income household, and use improve the efficiency of national medical insurance ${ }^{[18]}$. Potential strategies worth consideration include: simplifing the approval process of new drugs at home and abroad, improving their access to the market, reducing the innovation cost, and attracking more high-quality anti-cancer generic drugs to enter the market ${ }^{[31]}$. Importantly, in terms of the bidding and procurement process, National Health-care Security Administration negotiates with pharmaceutical manufacturers for reducing the OBs price and ensuring that the patients can afford the essential anticancer medicines ${ }^{[32]}$.

\section{Limitations}

In this survey, private hospitals and pharmacies were not included in the survey because they did not have the right to prescribe anti-cancer drugs. Therefore, the results may not represent the availability of anticancer medicines in all types of hospitals in Hubei Province. Second, during data collection, some "out-of-stock" medicines were not included in the calculation. Thus, the findings may not be generalizable to the whole availability of anticancer medicines. Lastly, for the convenience in comparing the affordability indicators, all medicines charges were calculated according to the course of 30 days. In fact, the patient's condition and tumor disease are different, which could lead to some biases in the accuracy of affordability. 


\section{Conclusion}

Compared with secondary hospitals, it is obvious that the availability of essential anticancer medicines was found to be much higher in tertiary hospitals. Overall, LPGs was prior to OBs in terms of availability. With the implementation of the Zero Mark-up Policy, the price of anticancer medicines returned to a reasonable level ${ }^{[29]}$, apart from some special Originator Brand medicines' price were still much higher than the international reference price. This study also identified several types of drugs that patients could hardly afford during the treatment. Policymakers should pay more attention to the possibility of poverty caused by expenditure on major cancer diseases. More actions should be adopted to regulate and restrict the pharmaceutical industry, drug circulation, patients and doctors, and improve the availability of essential anticancer medicines in Hubei Province.

\section{Abbreviations}

HAl

Health Action International ; WHO:World Health Organization; EML:Essential Medicine List; OB:Originator Brand; LPG:Lowest Priced Generic; NEML:National Essential Medicine List

\section{Declarations}

\section{Acknowledgement}

The authors were thankful for Professor Rui Huang and their stuff who support their available time for coordination and arrangement of investigation, in addition, we also would like to all the participants who attended in this study.

\section{Authors' contributions}

CYC participated in study design and conception, data acquisition, data analysis, manuscript drafting. We thank ZCF and YFD for guiding this study, and DF design the whole investigation and attend to collect data, ZQY and JW help us to input and check data, RXW and QF are responsible for proposing the revising suggestions of the manuscript draft.

\section{Funding}

This work was supported by National Natural Science Youth Fund (Grant Number: 71804052).

\section{Vailability of data and materials}

All data generated or analysed during this study are included in this published article.

\section{Ethics approval and consent to participate}


The study was approved by the Medical Ethics Committee of Tongji Medical College of Huazhong University of Science and Technology, and the approval number is 2020(S223). The Surveyed hospitals were informed by sending research letter prior to data collection, and all hospitals agreed to participant in this survey.

\section{Consent for publication}

Not applicable.

\section{Conflict of interest statement}

The authors have declared that no competing interests exist.

\section{Author details}

1.School of Medicine and Health Management, Tongji Medical College of Huazhong University of Science and Technology, Wuhan, Hubei, China.

2.Tongji Hospital Affiliated to Tongji Medical College of Huazhong University of Science and Technology, Wuhan, Hubei, China.

3.School of Pharmacy, Tongji Medical College of Huazhong University of Science and Technology, Wuhan, Hubei, China.

\section{References}

1. Zhu Y, Wang Y, Sun X, et al. Availability, Price and Affordability of Anticancer Medicines: Evidence from Two Cross-Sectional Surveys in the Jiangsu Province, China[J]. Int J Environ Res Public Health, 2019,16(19). doi:10.3390/ijerph16193728.

2. Erratum: Global cancer statistics 2018: GLOBOCAN estimates of incidence and mortality worldwide for 36 cancers in 185 countries[J]. CA Cancer J Clin, 2020,70(4):313. doi:10.3322/caac.21609.

3. Shaoqing L. The status quo and dilemma, hope and way out of cancer--Based on the analysis of the global cancer incidence, prevention and control situation in 2017 and 2018[J]. Medicine and Philosophy, 2019,40(12):20-23.

4. Smith SK, Nicolla J, Zafar SY. Bridging the gap between financial distress and available resources for patients with cancer: a qualitative study[J]. J Oncol Pract, 2014,10(5):e368-372.

doi:10.1200/JOP.2013.001342.

5. Hasan, S.S. ea. Pharmaceutical Policy Reforms to Regulate Drug Prices in the Asia Pacific Region: The Case of Australia, China, India, Malaysia, New Zealand, and South Korea[J]. Value Health Reg Issues, 2019,18:18-23.

6. Beran D, Ewen M, Lipska K, et al. Availability and Affordability of Essential Medicines: Implications for Global Diabetes Treatment[J]. Curr Diab Rep, 2018,18(8):48. doi:10.1007/s11892-018-1019-Z. 
7. Khanal S, Veerman L, Ewen M, et al. Availability, Price, and Affordability of Essential Medicines to Manage Noncommunicable Diseases: A National Survey From Nepal[J]. Inquiry, 2019,56:46958019887572. doi:10.1177/0046958019887572.

8. Dong Z, Tao Q, Sun G. Survey and analysis of the availability and affordability of essential drugs in Hefei based on WHO / HAI standard survey methods[J]. BMC Public Health, 2020,20(1):1405. doi:10.1186/s12889-020-09477-9.

9. Wu G, Gong S, Cai H, et al. The availability, price and affordability of essential antibacterials in Hubei province, China[J]. BMC Health Serv Res, 2018,18(1):1013. doi:10.1186/s12913-018-3835-x.

10. Liang Z, Qiuyun Z, Xuemeng Z. Investigation and Study on the Accessibility of Antibiotics in Essential Medicine List in Medical Institutions[J]. China Pharmacy, 2020,31(11):1281-1287. doi:10.6039/j.issn.1001-0408.2020.11.01.

11. Khuluza F, Heide L. Availability and affordability of antimalarial and antibiotic medicines in Malawi. PLoS One. 2017. 12(4): e0175399.

12. Statistical Yearbook of Hubei Province in 2019. Hubei Provincial Bureau of Statistics.,2019, available at: http://tjj.hubei.gov.cn/tjsj/sjkscx/tjnj/qstjnj/.

13. WHO Model List of Essential Medicines, 19th List 2019. World Health Organization.,2019, Available from: https://www.who.int/medicines/publications/essentialmedicines/EML_2015_FINAL_.

14. Saeed A, Saeed H, Saleem Z, et al. Evaluation of prices, availability and affordability of essential medicines in Lahore Division, Pakistan: A cross-sectional survey using WHO/HAl methodology[J]. PLoS One, 2019,14(4):e0216122. doi:10.1371/journal.pone.0216122.

15. Moye-Holz D, Ewen M, Dreser A, et al. Availability, prices, and affordability of selected essential cancer medicines in a middle-income country - the case of Mexico[J]. BMC Health Serv Res, 2020,20(1):424. doi:10.1186/s12913-020-05167-9.

16. Management Sciences for Health,International Drug Price Indicator Guide 2015,2019, Available from: https://mshpriceguide.org/en/download-files/.

17. Guan X, Hu H, Man C, Shi L. A survey of availability, price and affordability of essential medicines from 2011 to 2016 in Chinese secondary and tertiary hospitals. Int J Equity Health. 2018. 17(1): 158.

18. Sarwar MR, Iftikhar S, Saqib A. Availability of anticancer medicines in public and private sectors, and their affordability by low, middle and high-income class patients in Pakistan[J]. BMC Cancer, 2018,18(1):14. doi:10.1186/s12885-017-3980-3.

19. Khatib R MM, Shannon H CC, Rangarajan S TK, et al. Availability and affordability of cardiovascular disease medicines and their effect on use in high-income, middle-income, and low-income countries: An analysis of the PURE study data[J]. Lancet (London, England), 2015,387(61):9. doi:10.1016/S0140-6736(15)00469-9.

20. Lambojon K, Chang J, Saeed A. Prices, Availability and Affordability of Medicines with Value-Added Tax Exemption A Cross-Sectional Survey in the Philippines[J]. Int J Environ Res Public Health, 2020,17(14). 
21. Khuluza F, Haefele-Abah C. The availability, prices and affordability of essential medicines in Malawi: A cross-sectional study[J]. PLoS One, 2019,14(2):e0212125. doi:10.1371/journal.pone.0212125.

22. Rui $S$, Zhimei $X$, Yue L, et al. A Study on the Availability and Affordability of Anti-tumor Drugs in Public Hospitals-Based on the Data Analysis of the Typical Survey of Public Hospitals in Jiangsu Province[J]. Price theory and practice, 2019,(5):39-42.

23. Babar ZU, Ramzan S, El-Dahiyat F, et al. The Availability, Pricing, and Affordability of Essential Diabetes Medicines in 17 Low-, Middle-, and High-Income Countries[J]. Front Pharmacol, 2019,10:1375. doi:10.3389/fphar.2019.01375.

24. Abdel Rida N, Mohamed Ibrahim MI, Babar Z. Relationship between pharmaceutical pricing strategies with price, availability, and affordability of cardiovascular disease medicines: surveys in Qatar and Lebanon[J]. BMC Health Serv Res, 2019,19(1):973. doi:10.1186/s12913-019-4828-0.

25. Dorj G, Sunderland B, Sanjjav T, et al. Availability, affordability and costs of pediatric medicines in Mongolia[J]. BMC Pediatr, 2018,18(1):149. doi:10.1186/s12887-018-1123-X.

26. Teng JP, Yang ZY, Zhu YM, et al. Gemcitabine and cisplatin for treatment of lung cancer in vitro and vivo[J]. Eur Rev Med Pharmacol Sci, 2018,22(12):3819-3825. doi:10.26355/eurrev_201806_15266.

27. Yahong L. Study on Family Burden, Quality of Life and Influencing Factors of Lung Cancer Patients in a Cancer Hospital:[D].Lan Zhou University,2019.

28. Cameron A, Ewen M, Ross-Degnan D, et al. Medicine prices, availability, and affordability in 36 developing and middle-income countries: a secondary analysis[J]. Lancet, 2009,373(9659):240-249. doi:10.1016/S0140-6736(08)61762-6.

29. Mao W, Huang Y, Chen W. An analysis on rational use and affordability of medicine after the implementation of National Essential Medicines Policy and Zero Mark-up Policy in Hangzhou, China[J]. PLoS One, 2019,14(3):e0213638. doi:10.1371/journal.pone.0213638.

30. Faruqui N, Martiniuk A, Sharma A, et al. Evaluating access to essential medicines for treating childhood cancers: a medicines availability, price and affordability study in New Delhi, India[J]. BMJ Glob Health, 2019,4(2):e001379. doi:10.1136/bmjgh-2018-001379.

31. Eden T, Burns E, Freccero P. Are essential medicines available, reliable and affordable in lowmiddle[J]. J Cancer Policy, 2018. doi:10.1016/j.jcpo.2018.12.001.

32. Jiao Y, Yangzheng L, Lin Y. Evaluation of the bid-winning effect of centralized procurement of medicines in public hospitals in 6 central and southern provinces or districts[J]. China Pharmacy, 2018,29(15):2017-2020.

\section{Figures}




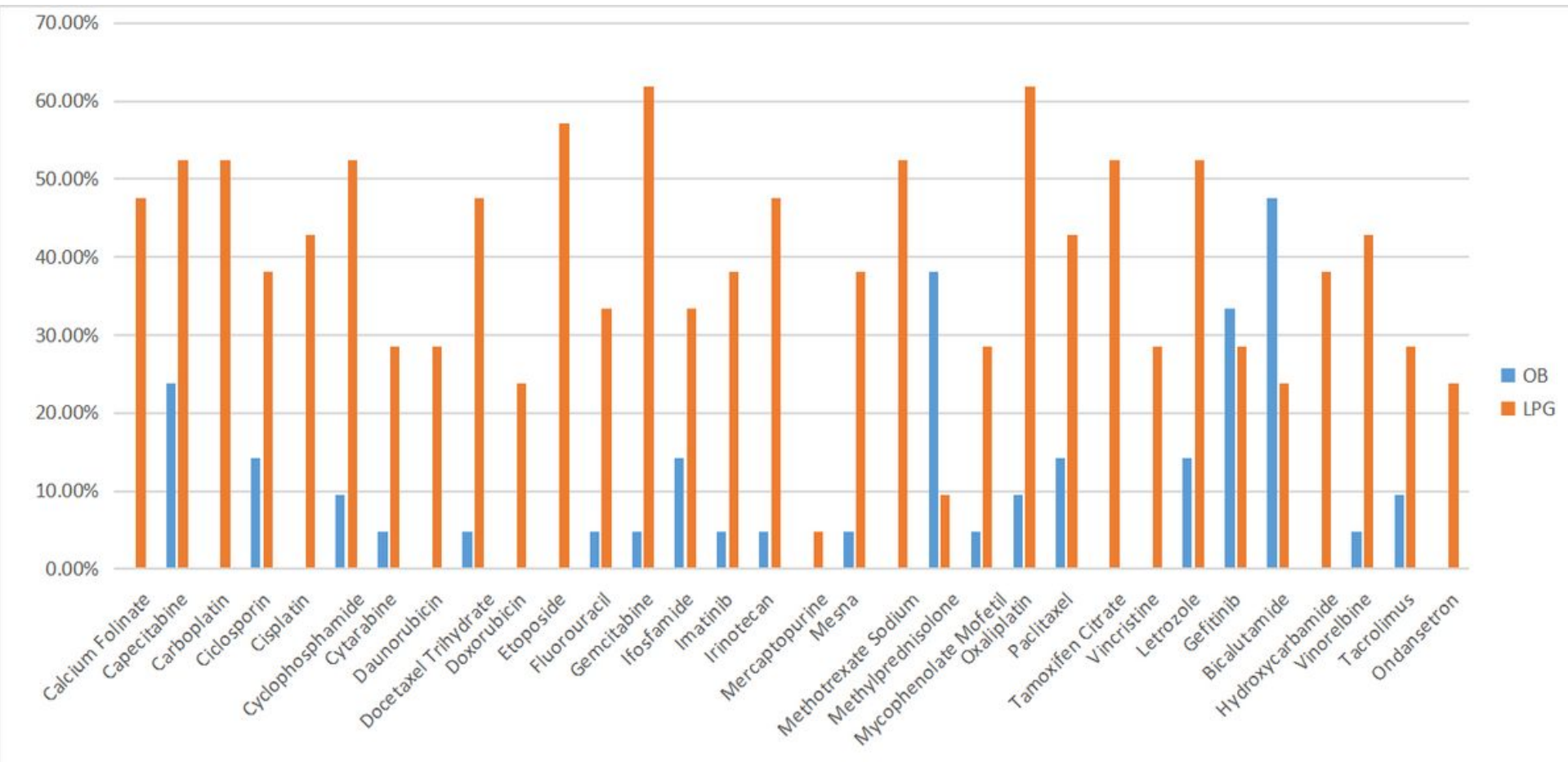

\section{Figure 1}

The availability of LPG and OB medicines in tertiary hospital

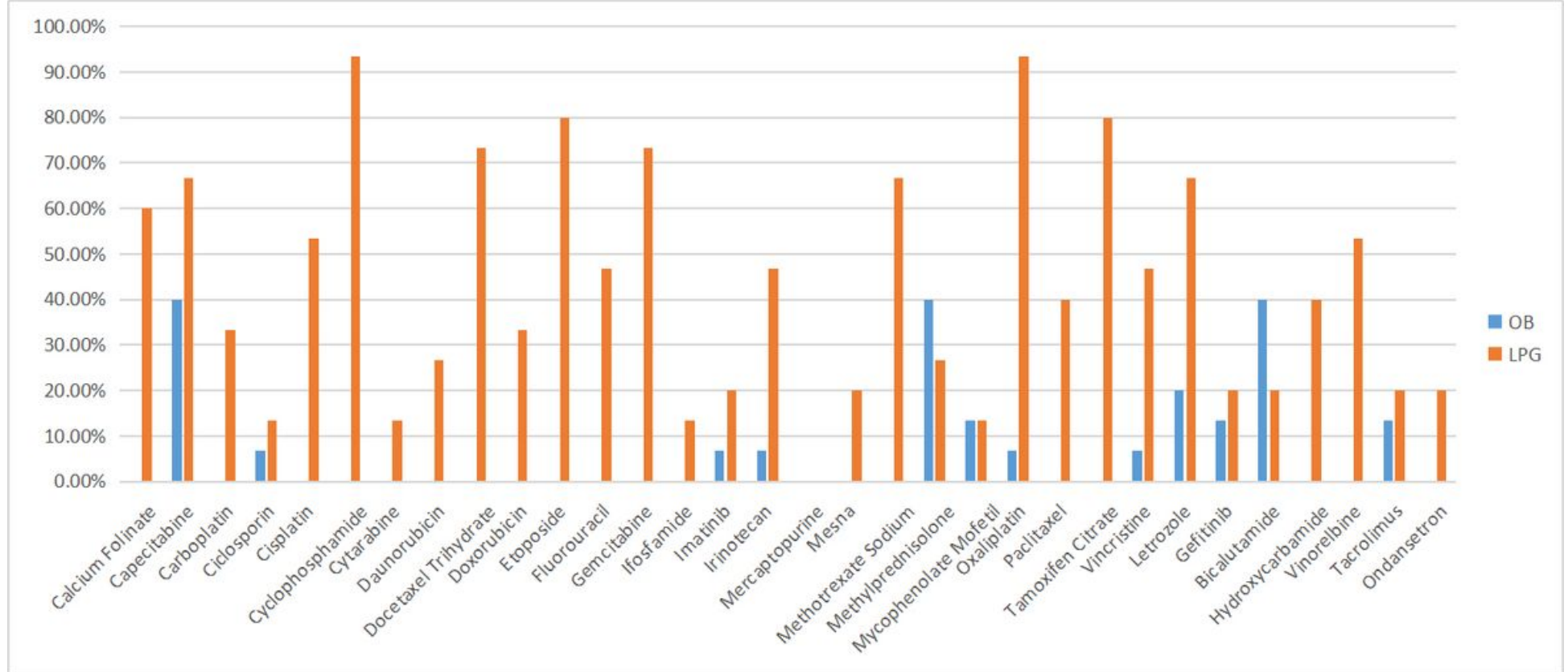

Figure 2

The availability of LPG and OB medicines in secondary hospital 

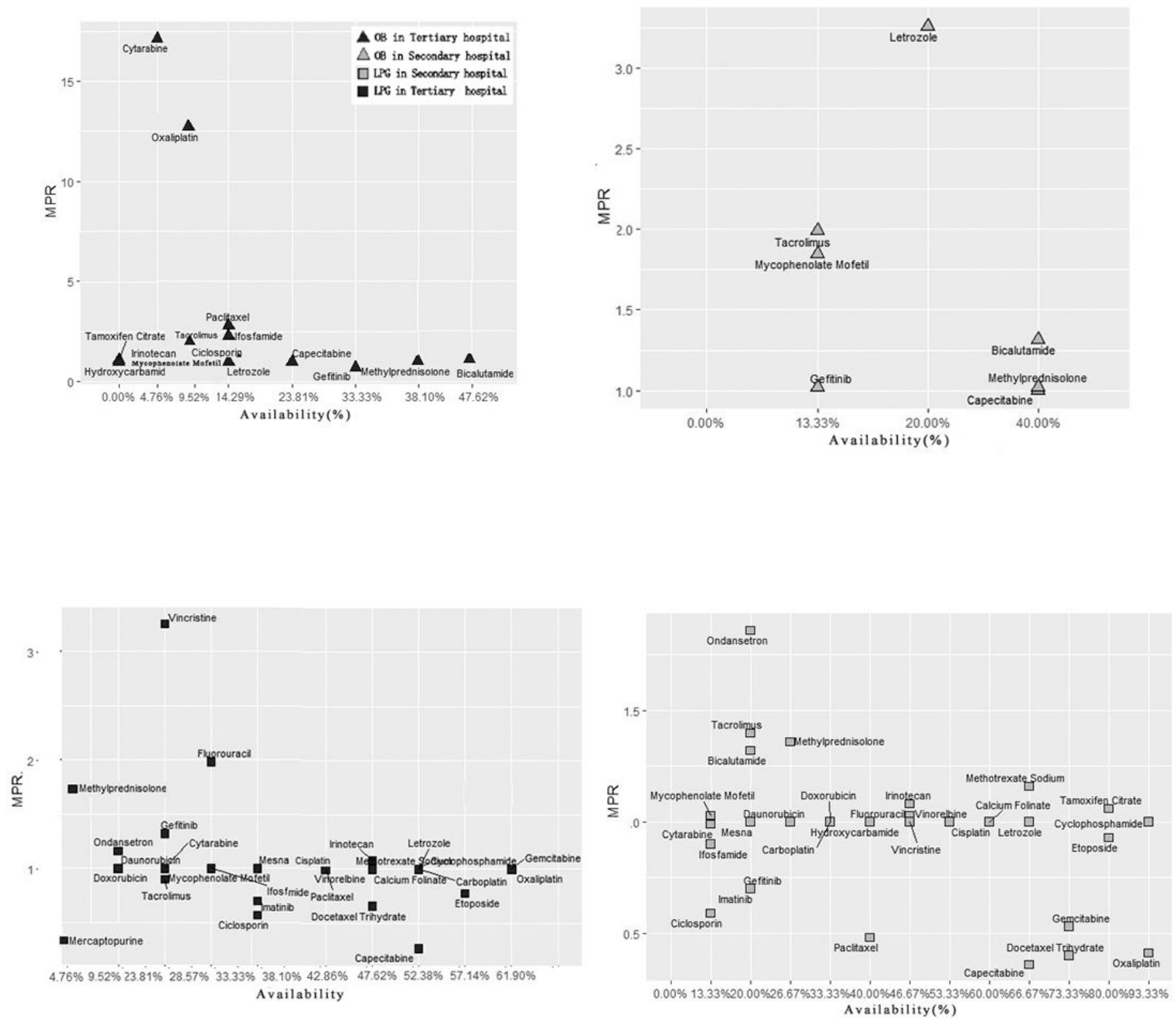

\section{Figure 3}

Comprehensive analysis of anticancer medicine availability and MPR in Hubei Province.(a) Scatter plot of OBs availability and MPR in tertiary hospitals. (b) Scatter plot of OBs availability and MPR in secondary hospitals. (c) Scatter plot of LPGs availability and MPR in tertiary hospitals. (d) Scatter plot of LPGs availability and MPR in secondary hospitals. 


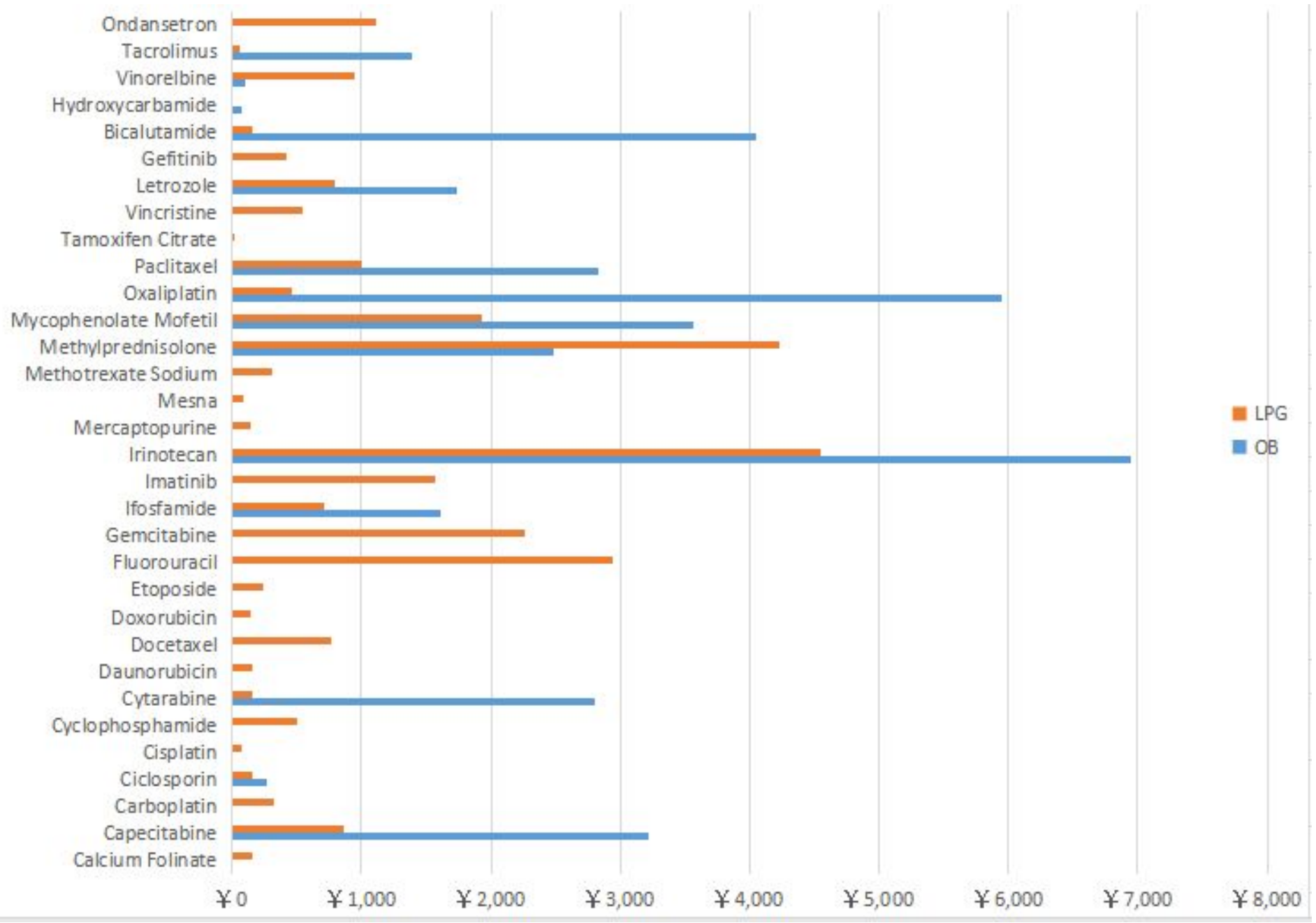

\section{Figure 4}

Total treatment cost of 32 anti-neoplastic for Originator Brand and Lowest Prices Generic expressed in RMB across Hubei Province 